\title{
Similar survival outcome after endoscopic and open approaches for sinonasal mucosal melanoma*
}

\author{
M. Lundberg', A. Haapaniemi', J. Hagström², S. Juteau², M. Hernberg³, \\ A.A. Mäkitie ${ }^{1,4}$, S.I. Vento ${ }^{1}$ \\ 'Department of Otorhinolaryngology - Head and Neck Surgery, University of Helsinki and Helsinki University Hospital, Helsinki, \\ Finland \\ 2 Department of Pathology, University of Helsinki and Helsinki University Hospital, Helsinki, Finland \\ ${ }^{3}$ Department of Oncology, Comprehensive Cancer Centre, Helsinki University Hospital and University of Helsinki, Helsinki, Finland \\ ${ }^{4}$ Division of Ear, Nose and Throat Diseases, Department of Clinical Sciences, Intervention and Technology, Karolinska Institutet and \\ Karolinska University Hospital, Stockholm, Sweden
}

Rhinology 57: 2, $132-138,2019$

https://doi.org/10.4193/Rhin18.123

*Received for publication:

June 23, 2018

Accepted: September 3, 2018

Background: To describe a cohort of sinonasal mucosal melanoma (SNMM) patients, and to assess if choice of surgical approach (open versus endoscopic) has impact on survival.

Methodology: Adequate data on clinical presentation, treatment, and recurrence pattern were available for 58 consecutive patients treated for SNMM at the Helsinki University Hospital (HUH) between 1983 and 2016.

Results: The 5-year disease-specific survival (DSS) was $27 \%$ and overall survival $25 \%$ for the whole cohort. The 3-year DSS for patients treated with curative intent with endoscopic surgery was comparable to open surgery ( $56 \%$ and $51 \%$, respectively). Patients with tumours arising from the paranasal sinuses and patients with Stage IV disease had significantly worse prognosis compared with other locations and Stage III patients. All patients who had disease persistence at three months after primary treatment succumbed to SNMM. Post-operative radiotherapy did not affect survival significantly, but a trend towards improved local control was observed.

Conclusions: Local control after endoscopic surgery was comparable to open surgery. Small tumours without local or locoregional spread had improved prognosis, independent of surgical approach. Disease persistence after treatment with curative intent led to death invariably.

Key words: head and neck neoplasms, minimally invasive, nose, paranasal sinuses, radiotherapy

\section{Introduction}

Sinonasal cancers (SNC) are rare and account for $3 \%$ of all cancers in the head and neck region. The majority of them are squamous cell carcinomas, whereas melanomas comprise less than $4 \%{ }^{(1)}$. Sinonasal mucosal melanomas (SNMMs) account for less than $1 \%$ of all melanomas and $4 \%$ of head and neck melanomas $(1,2)$. The annual age-standardized incidence rate is increasing and is in Sweden estimated to be 1.08 and 0.67 per one million women and men, respectively ${ }^{(3)}$.

SNMM carries a worse prognosis than many other types of SNCs. The 5 -year disease-free survival typically ranges from 12 to
$44 \%$ due to its aggressive behavior, tendency for early distant metastasis and high incidence of local recurrences ${ }^{(2,4,5)}$. SNMM is mostly diagnosed at an advanced stage, which decreases survival. Symptoms can be mixed with benign processes or alternatively, patients can be asymptomatic, which cause both patient- and diagnostic delay. Melanoma is not considered radiosensitive ${ }^{(5,6)}$ and thus, radical surgery with clear margins is currently the therapy of choice ${ }^{(2)}$. However, postoperative radiotherapy may increase local control ${ }^{(7,8)}$. Traditionally, surgery has been performed with open approach, but endoscopic surgery has gained popularity due to lower morbidity and complication 
rates associated with this technique ${ }^{(2,9)}$. Endoscopic approach carries a similar or, possibly, improved outcome ${ }^{(5,10)}$. In this study, we present a retrospective, single-center series of 58 SNMM patients with a focus on surgical approach and survival.

\section{Patients and methods}

\section{Patients}

We retrospectively reviewed all patients treated for SNMM between 1983 and 2016 at the Helsinki University Hospital (HUH), Finland. HUH is a tertiary-care academic center with a referral area of 1.6 million people, which corresponds to almost one third of the nation-wide population. Almost half of Finland's SNMM patients were treated at $\mathrm{HUH}$ during this period ${ }^{(11)}$. Patients were identified based on the ICD-codes C30, and C31 (malignant neoplasm of the nasal cavity / paranasal sinuses) from the hospital and Department of Pathology registries, and from the Finnish Cancer Registry. Adequate data on clinical presentation, treatment, and recurrence pattern were available for 58 consecutive patients. All patients had a newly diagnosed SNMM and their treatment strategy was planned at a multidisciplinary tumour board meeting. Patients were staged according to the 7th UICC staging system that categorizes tumours into Stage III (T3N0; tumour limited to mucosa), Stage IVA (T3N+ or T4aN0; tumour invades deep soft tissue, cartilage, bone or overlying skin), Stage IVB (T4bNO/N+; tumour invades the brain, skull base, cranial nerves, or carotid artery) and Stage IVC (any tumour with distant metastasis).

\section{Statistical analyses}

Statistical analyses were performed with the SPSS 22.0 statistical package (IBM Corp. Armonk, NY, USA). Categorical data were compared using the chi-squared test. Survival figures were calculated with the Kaplan-Meier product limit estimate method and significance of differences between groups was investigated with the log-rank test. Survival was calculated from the date of diagnosis to the date of the event (recurrence, death, last follow-up). For overall survival (OS), death from any cause was recorded as an event, and for disease-specific survival (DSS) only death from SNMM was recorded as an event. For local control (LC), local recurrence was recorded as an event. Causes of death were obtained from Statistics Finland. The shortest follow-up until death was one month. Of the patients alive, follow-up in our hospital ranged between 8 and 77 months. In three cases follow-up continued in regional hospitals after 2 to 13 months of follow-up in our hospital. All these patients were, according to data from Statistics Finland, alive more than two years after treatment. A p-value $<0.05$ was considered statistically significant. An institutional research approval was granted for the study.

\section{Results}

The median age of the 58 SNMM patients was 75 years (range,
Table 1. Characteristics of tumours and patients $(n=58)$, and comparison of the endoscopic and open resection cohorts $(n=54)$.

\begin{tabular}{|c|c|c|c|c|}
\hline Characteristic & $\begin{array}{c}\text { Total } \\
n=58 \\
(100 \%)\end{array}$ & $\begin{array}{c}\text { Open } \\
\text { surgery } \\
n=36 \\
(62 \%)\end{array}$ & $\begin{array}{c}\text { Endo- } \\
\text { scopic } \\
\text { surgery } \\
n=18 \\
(31 \%)\end{array}$ & p-value* \\
\hline $\begin{array}{l}\text { Gender } \\
\text { Female } \\
\text { Male }\end{array}$ & $\begin{array}{l}31(53) \\
27(47)\end{array}$ & $\begin{array}{l}21(36) \\
15(26)\end{array}$ & $\begin{array}{c}8(14) \\
10(17)\end{array}$ & 0.622 \\
\hline Age, mean (yrs) & $\begin{array}{c}75 \\
(43-95)\end{array}$ & $\begin{array}{c}69 \\
(43-94)\end{array}$ & $\begin{array}{c}78 \\
(51-92)\end{array}$ & 0.012 \\
\hline $\begin{array}{l}\text { T class } \\
\text { T3 } \\
\text { T4a } \\
\text { T4b } \\
\text { Unknown }\end{array}$ & $\begin{array}{c}29(50) \\
16(28) \\
8(14) \\
5(9)\end{array}$ & $\begin{array}{c}20(35) \\
10(17) \\
2(3) \\
4(7)\end{array}$ & $\begin{array}{c}8(14) \\
4(7) \\
6(10) \\
0(0)\end{array}$ & 0.060 \\
\hline $\begin{array}{l}\text { N class } \\
0 \\
1 \\
\text { Unknown }\end{array}$ & $\begin{array}{c}49(85) \\
4(7) \\
5(9)\end{array}$ & $\begin{array}{c}32(55) \\
0(0) \\
4(7)\end{array}$ & $\begin{array}{c}15(26) \\
3(5) \\
0(0)\end{array}$ & 0.032 \\
\hline $\begin{array}{l}\text { Primary location } \\
\text { Nasal cavity } \\
\quad \text { (undefined) } \\
\text { Septum } \\
\text { Nasal floor } \\
\text { Nasal lateral wall } \\
\text { Vestibule } \\
\text { Maxillary sinus } \\
\text { Ethmoid sinus }\end{array}$ & $\begin{array}{c}16(28) \\
9(16) \\
3(5) \\
15(26) \\
2(3) \\
9(16) \\
4(7)\end{array}$ & $\begin{array}{c}11(19) \\
7(12) \\
1(2) \\
10(17) \\
1(2) \\
5(9) \\
1(2)\end{array}$ & $\begin{array}{l}3(5) \\
1(2) \\
2(3) \\
5(9) \\
1(2) \\
3(5) \\
3(5)\end{array}$ & 0.556 \\
\hline $\begin{array}{l}\text { WHO performance } \\
\text { status } \\
0 \\
1 \\
2 \\
3 \\
4 \\
\text { Unknown }\end{array}$ & $\begin{array}{c}23(40) \\
9(16) \\
14(24) \\
2(3) \\
5(9) \\
5(9)\end{array}$ & $\begin{array}{c}15(26) \\
7(12) \\
8(14) \\
1(2) \\
1(2) \\
4(7)\end{array}$ & $\begin{array}{c}7(13) \\
2(4) \\
6(10) \\
1(2) \\
2(3) \\
0(0)\end{array}$ & 0.062 \\
\hline
\end{tabular}

*p-value comparing open and endoscopic resection groups.

43-95) and a slight majority (53\%) were women. Detailed patient and tumour characteristics are listed in Table 1. On average, the patients had suffered from nasal symptoms for four months (range, 1-12). The most common symptoms were epistaxis (75\%), nasal obstruction (73\%) and nasal discharge (39\%).

Ophthalmologic (17\%) and neurologic symptoms (4\%) were also observed. The most common primary tumour location was nasal cavity (78\%) and mainly its lateral wall. Twenty-eight percent of the patients with tumours in the nasal cavity had wide tumour extension hindering precise assessment of the primary site. Of the patients with wide tumour extension, 31\% had tumour spread to adjacent vital structures, such as the orbit or skull base. Wide local spread was also common in ethmoidal tumours (75\%) and tumours in maxillary sinus (56\%). No melanomas originated from other paranasal sinuses.

The TNM class could not be defined retrospectively for five 


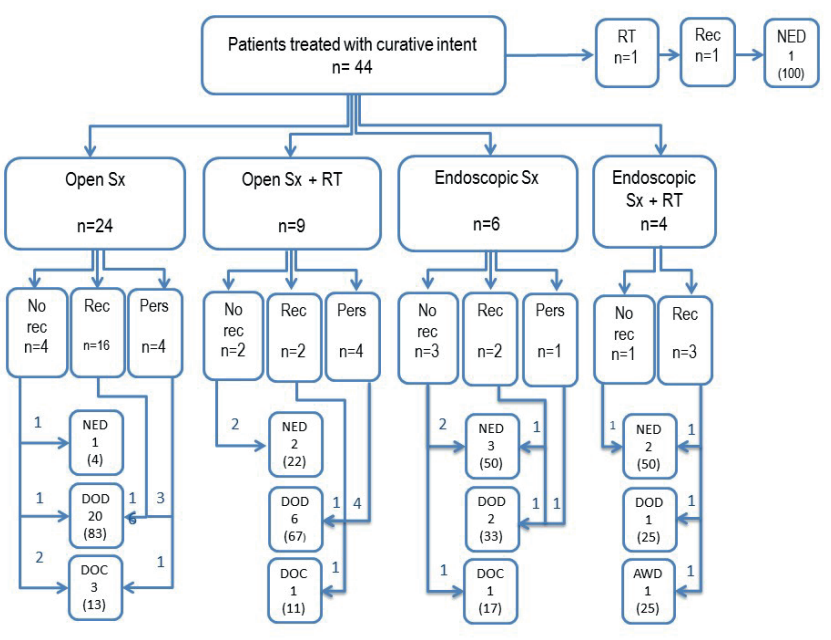

Figure 1. Surgical treatment cohorts and outcome graph. Outcome percentage defined within the particular treatment arm. Sx = Surgery, $\mathrm{RT}=$ Radiotherapy, $\mathrm{Rec}=$ Recurrence, No rec $=$ No recurrence, Pers $=$ Persisting disesase, NED = No evidence of disease, $\mathrm{DOD}=$ Dead of disease, $\mathrm{DOC}=$ Dead of other cause, $\mathrm{AWD}=$ Alive with disease.

patients. Regional nodal spread was uncommon; only four (8\%) patients had nodal metastasis at presentation. It was, however, more common among the 24 patients with T4 tumours ( $n=3$; $13 \%)$ than among the 29 with T3 tumours ( $n=1 ; 4 \%)$, although this difference was not statistically significant $(p=0.32)$. Primary stage distant metastases were present in four patients (8\%) and none of them had regional metastasis. All primary stage distant metastases were detected in patients presenting with SNMM after the year 2000. In this series preoperative radiologic staging before the year 1997 only included a chest x-ray, occasionally combined with abdominal ultrasound examination. Consequently, distant metastases might have been overlooked. Since 1997 computed tomography and/or magnetic resonance imaging has been in routine use for staging purposes at the $\mathrm{HUH}$. The patient and tumour parameters in the cohort with open surgical approach were comparable to those of the endoscopic group in all other aspects except for mean age, as patients selected for endoscopic surgery were older (mean age: 78 vs. 69 yrs, $\mathrm{p}=0.012$, Table 1).

\section{Treatment}

\section{Treatment with curative intent}

For 44 patients $(76 \%)$ the treatment was administered with curative intent. Patients younger than 70 years were more likely to undergo curatively aimed treatment compared with older patients ( $95 \%$ vs. $67 \% ; p=0.023)$. Thirty $(68 \%)$ out of the patients with curative treatment intent had surgery alone and 13 (30\%) had surgery with postoperative radiotherapy (RT). One patient was unconventially treated with RT alone to a total dose of 60 Gy in 2 Gy fractions due to multifocal, inoperable, submucosal

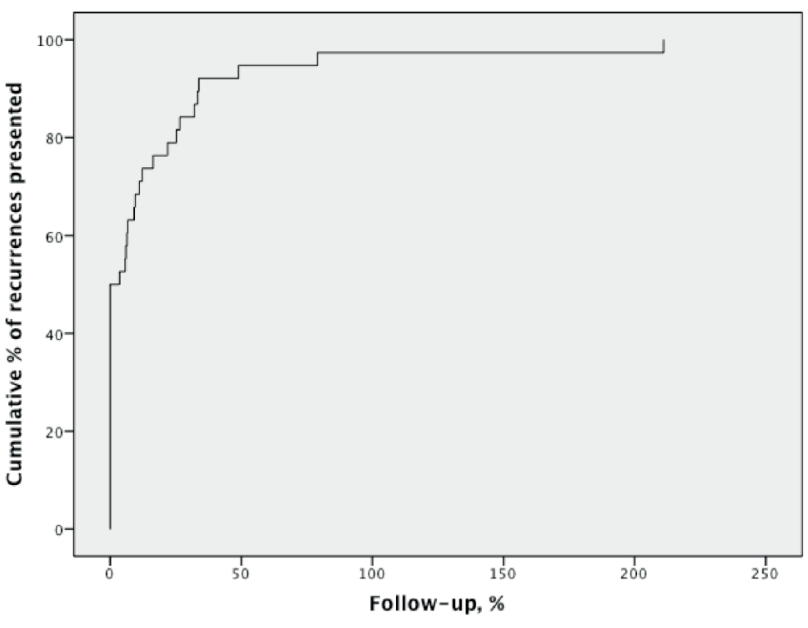

Figure 2. The majority of recurrences (88\%) occurred within 3 years after treatment with curative intent.

disease. The tumour recurred locally after 12 months and the patient developed liver metastasis. Recurrent disease was successfully treated with sinonasal debulking and a combination of docetaxel, carboplatine and liver surgery.

Ten (25\%) out of the 40 resections with curative intent were performed with endoscopic technique, all during this millennium (Figure 1). Two of these ten patients had a T4 tumour. Characteristics of both the endoscopic and open resection cohorts are described in Table 1.

No patients were treated primarily with systemic therapy. One patient, who was diagnosed with disseminated disease postoperatively in the primary surgery with curative intent, received, as first-line therapy, anti-PD1-antibody therapy (pembrolizumab), and has since then remained disease-free for 24 months.

\section{Treatment with palliative intent}

Treatment was given with palliative intent for 14 patients (24\%). All of these patients had class T4 tumours and either distant metastasis or extensive local/regional disease hindering curatively aimed treatment. Eleven patients had either surgical debulking or radiofrequency ablation (RFA), which was followed by RT for four patients. Eight (73\%) of these debulking procedures were performed endoscopically or with endoscopic assistance. Five of them were performed before 2012 when endoscopic surgery became the primary surgical technique for SNMM at HUH. One patient received RT only and two patients received only systemic therapy.

\section{Outcome}

The median follow-up time for all patients was 16 months (mean, 38; range, 1-229), and for the surviving patients, 33 months (mean, 42; range, 2-93). Of the 44 patients treated with curative intent, ten (23\%) were alive at the end of follow-up, and 


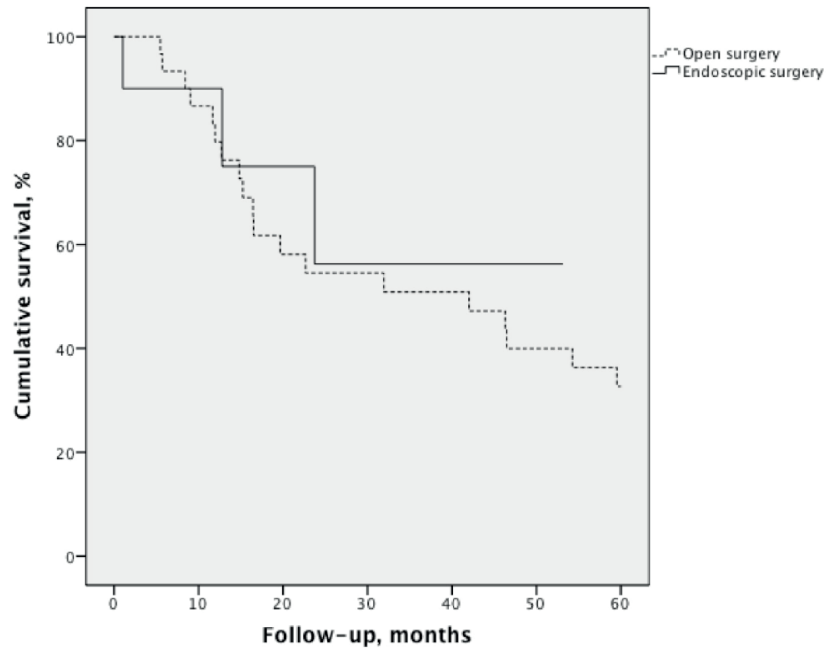

Figure 3. Disease-specific patient survival for open approach versus endoscopic approach.

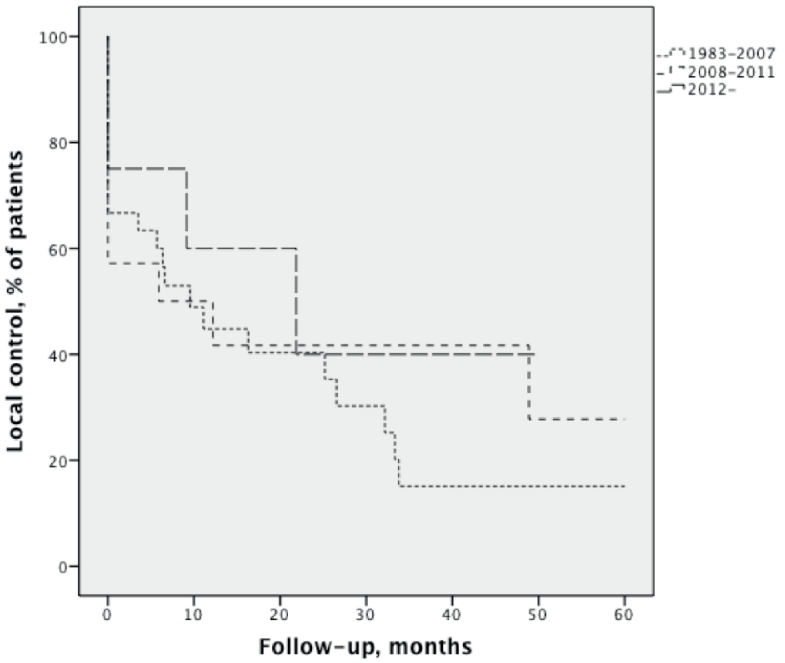

Figure 4. Local control stratified according to main operative approach: open approach during 1983-2007, mainly open approach with endoscopy for small resections during 2008-2011, endoscopy as primary approach 2012-2016. one of them had persistent disease. At three months after primary treatment 33 (75\%) were disease-free but 24 of them had a recurrence later. The median time to recurrence after treatment was 13 months (range, 4-211), and $88 \%$ of them occurred within 3 years after treatment completion (Figure 2). Of the 24 recurrences, $67 \%(n=16)$ were local, $21 \%(n=5)$ distant, and $13 \%$ $(n=3)$ both local and distant. Local recurrences were treated with curative intent in $75 \%(n=12)$ of cases. Eight of these patients died of SNMM and one died of other causes, but with persistent disease. Three patients with a recurrence were disease-free at the end of follow-up. In one patient a locoreginal metastasis was removed by neck dissection. The follow-up continued after 13 months in another hospital, but according to Statistics Finland, the patient was alive seven years later. The second patient had local re-resection and was disease-free after 77 months, and the third patient was treated as described above with local resection, chemotherapy and liver resection and was 28 months disease-free at the end of study.

All patients $(n=9)$ treated with curative treatment intent with disease persistence at three months after treatment, died: eight of them of SNMM and one of intercurrent disease. In five (56\%) of the nine patients with persistent disease at three months, the disease had disseminated. Outcome data are shown in Figure 1.

\section{Survival}

The 5 -year DSS was $27 \%$ and OS $25 \%$ for the whole cohort. The respective 10-year DSS and OS figures were $16 \%$ and $14 \%$. Gender did not affect DSS. Patients under 70 years of age had a better 5-year DSS (50\%) compared with older patients (15\%; $p=0.036)$. Patients with tumours arising from the nasal cavity had better DSS ( 31 vs. $15 \%$; $p=0.021$ ) and OS ( $29 \%$ vs. $15 \%$; $\mathrm{p}=0.038$ ) compared with patients with tumours arising in the maxillary and ethmoidal sinuses. For patients with Stage III disease the 5 -year DSS was $45 \%$ whereas for Stage IV patients it was significantly lower (14\%, $\mathrm{p}=0.001)$. The OS for these groups was only slightly lower, as expected: for Stage III patients $41 \%$ compared to $14 \%$ for Stage IV patients ( $p=0.003)$. Patients with T3 tumours had a significantly improved DSS compared to T4 tumours ( $39 \%$ vs. $14 \% ; p=0.006$ ). The difference in DSS was even clearer when stratified by N class; the 5 -year DSS was $32 \%$ for patients with $\mathrm{N} 0$ neck but $0 \%$ for the $\mathrm{N}+$ patients, respectively $(\mathrm{p}<0.001)$. The OS rate for $\mathrm{NO}$ and $\mathrm{N}+$ disease was $30 \%$ and $0 \%$, respectively $(\mathrm{p}<0.001)$. Two of the $\mathrm{N}+$ patients were treated with curative intent. One patient died of intercurrent disease shortly after surgery, and the other developed distant metastases three months after treatment.

Endoscopic approach with curative intent has been the primary treatment approach at our department since 2012; during 20082012 it was an alternative surgical approach for small tumours. Before this time period, endoscopic technique was used only for palliative debulking. In the present material, $28 \%$ of T3 and $15 \%$ of T4 patients were treated with curative intent by either endoscopic approach alone or combined with post-operative RT. The median follow-up time for patients treated with curative intent by endoscopic surgery was 17 months (mean 20; range, 1-53). The 3-year DSS for patients treated with endoscopic surgery with curative intent was comparable to open surgery; endoscopic surgery $56 \%$ vs. open surgery $51 \%$ ( $p=$ n.s., Figure 3 ). Similarly, the 3-year LC rate did not significantly differ between endoscopic (36\%) and open surgery (31\%). Stratification of 
patients according to $T$ class did not produce any significant differences in survival and LC figures between endoscopic and open surgery groups. When stratifying into three groups, according to the use of endoscopic approach; not in use during 1983-2007 ( $\mathrm{n}=31$ ), only for small resections during 2008-2011 $(\mathrm{n}=15)$, and as the primary approach during 2012-2016 $(\mathrm{n}=12)$ DSS improved from $20 \%$ to $35 \%$, and $49 \%$, respectively ( $p=n . s$.). The median duration of LC showed an improvement over time $9.6 \pm$ SE 3.8 months (1983-2007); $5.9 \pm$ SE 10.5 (2008-2011), and $21.8 \pm$ SE 12.6 months (2012-2016) (Figure 4).

Fourteen patients treated with curative intent received RT, 13 of them combined with surgery. Data on RT dose were available for 11 patients, 5 (45\%) of who received a minimum dose of $60 \mathrm{~Gy}$. The mean RT dose, when administered with curative intent, was 53 Gy (range, 38-66), and mainly administered in 2 Gy fractions. The 38 Gy dose remained low due to RT complications. Postoperative RT for patients treated with curative treatment intent did not significantly affect the 5-year OS (RT+ 51\%, RT- 27\%; $\mathrm{p}=$ n.s.) or DSS (RT+ 51\%, RT- 29\%, p=n.s.). However, a trend towards a slightly better 5-year LC was observed in patients treated with post-operative RT (28\%) compared with those who did not undergo RT (22\%; $p=0.060)$. When administered with palliative intent, the RT doses varied between 17 and $60 \mathrm{~Gy}$, the high dose aiming for local control in a patient with distant metastasis.

SNMM recurrence and outcome based on surgical approach and RT are shown in Figure 1. At the end of follow-up, $4 \%$ of patients treated with open surgery alone showed no evidence of disease. When open surgery was combined with RT $22 \%$ of patients were disease-free, whereas after endoscopic surgery, with or without RT, $50 \%$ of the patients showed no evidence of disease.

\section{Discussion}

We present data on a population-based series of 58 SNMM patients treated at a single tertiary care university hospital during a 33-year period with a focus on survival after endoscopic surgery. Ten patients were managed with curative intent with primary endoscopic surgery and all these during the latter time period i.e. during this millenium. Similar to other studies, no significant differences were detected between open and endoscopic surgery regarding DSS or OS ${ }^{(12,13)}$. Tumour size caused no group bias. We were not able to verify the improved local control for patients treated with endoscopic surgery shown by Lund et al. and Miglani et al., possibly due to the short mean follow-up for endoscopically treated patients ${ }^{(5,10,14)}$. The mean LC time and the percentage of disease-free patients, however, showed a trend towards improvement after endoscopic resections were introduced at our institution. In accordance with other studies, we found tumour stage and site to be prognostic ${ }^{(4,15-18)}$, independent of surgical approach. As recurrence is devastating for the prognosis, radical primary surgery remains essential. This might be more easily achieved in smaller tumours in the lower nasal cavity than in the paranasal cavities that are situated closer to vital structures such as the skull base. Another possible explanation is that tumours in the nasal cavity are diagnosed earlier. However, in this retrospective material, time from the onset of symptoms to the diagnosis was similar for different tumour localizations. It is likely that paranasal sinus SNMMs, carrying 5-year OS rates of $0-11 \%$, cause symptoms mainly at a later stage or are more aggressive by nature.

Regardless of various treatment approaches presented in the existing reports, the prognosis of SNMM has generally remained poor. The 5 -year DSS rate of $24 \%$ observed for the current cohort is in line with that reported from other centers ${ }^{(3,5,6)}$. Higher 5 -year DSS rates reaching up to $32 \%$ have been reported in a US cohort, but the series also included oral and pharyngeal sites that generally carry a better prognosis ${ }^{(16)}$. Disseminated disease is common in SNMM and although all distant metastases in the present study were diagnosed after the year 2000, we believe that this is rather a sign of improved diagnostics than of changed tumour behavior. SNMM is known for its aggressiveness with a high risk of early local recurrences. In this study $60 \%$ of the patients treated with curative intent had a local recurrence, and only three had long-term survival after salvage treatment. This emphasizes the need for an early complete primary resection, which is generally accepted as the best treatment approach for SNMM ${ }^{(2)}$. RT alone is not recommended as primary treatment

(2), but there is increasing evidence that locoregional control can be improved with the addition of adjuvant RT, administered with new techniques such as intensity-modulated $\mathrm{RT}(7,8,19,20)$. In the present series RT was administered postoperatively to 17 patients, four of whom received it as part of palliative treatment to increase local control. Only five out of the 16 patients (31\%) having local recurrence had received adjuvant RT. We observed a trend towards minor improvement in LC for postoperative RT, but in accordance with other studies we found no effect of RT on survival ${ }^{(15,19)}$.

Although SNMM does not primarily spread to regional lymph nodes, lymphatic spread has been reported as a negative prognostic marker ${ }^{(5,16)}$. Although neck dissections were performed for the patients with $\mathrm{N}+$ disease, none of them remained disease-free at three months. In agreement with previous studies, nodal spread, although uncommon, seems to be a relevant prognostic factor ${ }^{(5)}$.

The main limitation of this study is its retrospective nature and the long study period, during which diagnostic workup, surgical methods, and treatment protocols have changed. These changes cause difficulty in data interpretation and might render a bias regarding the improved disease control over time. We 
were not able to find enough data on surgical margins, tumour markers or genetic mutation status in our cohort to carry out any informative statistical analyzes and to investigate the role predictive or prognostic role of these factors. Since 2009 the 7 th edition of UICC has been in use and we were compelled to re-stage older cases in this series based on medical records; a task that can occasionally be challenging. However, this study involves a rather large patient series compared with many previous reports on SNMM and represents a single-center experience with a limited number of surgeons having been responsible for the surgery. Data on follow-up, recurrences and cause of death are also highly accurate. In a rare disease like SNMM, multicenter prospective treatment trials would be ideal. While waiting for such studies, it is important to report on results of retrospective patient series to add to the existing data for meta-analyses.

Compared with open surgery, the endoscopic approach offers a superior view of the surgical field, facilitating improved tumour margin assessment ${ }^{(14)}$. The current study demonstrates that endoscopic surgery and open surgery will result in similar survival $(10,12,13,21)$. However, morbidity and complication rates are lower for endoscopic surgery, which makes this approach an attractive alternative when combined with shorter operative time and hospital stay, and improved cosmetic outcome ${ }^{(2,12,13,21)}$.

With respect to the high rate of local recurrences postoperative RT needs to be considered if sufficient pathological margins cannot be verified. Modern treatment strategy should also include immunohistochemical and molecular pathological analysis of the tumour. At least immunohistochemical analysis of PD-L1 and BRAFV600E is relevant ${ }^{(22)}$. Moreover, NGS-sequencing with a suitable panel detecting at least KIT and NRAS mutations is advisable, to be able to plan oncological systemic treatment for recurrent disease ${ }^{(23)}$. Adjuvant anti-PD1-antibody treatment should also be considered for this group of patients in order to improve the poor prognosis ${ }^{(24-27)}$.

\section{Conclusion}

This retrospective study on 58 SNMM patients provides further evidence on endoscopic surgery offering equal rates of local control and survival compared with open approach. Early diagnosis and treatment onset as well as achieving negative surgical margins are of utmost importance in the management of this aggressive disease. T3 tumours without locoregional or distant spread have an increased chance of survival compared with more extensive tumours.

\section{Authorship contribution}

The contribution to the planning and design was done by SV and AM. Acquisition of data was made by ML, AH, JH and SV. The interpretation and analysis of data was made by $M L, A H$, and SV. Article drafting and revising was made by all authors.

\section{Conflict of interest}

The authors have no conflicts of interest to disclose.

\section{References}

1. El-Naggar AK, Chan JK, Rubin Grandis J, Takata T, Slootweg PJ. World Health Organization Classification of Tumours, Pathology and Genetics of Head and Neck Tumours. 2017 International Agency for Research on Cancer (IARC), Lyon.

2. Lund VJ, Stammberger $H$, Nicolai $P$, Castelnuovo P, Beal T, Beham A, et al. European position paper on endoscopic management of tumours of the nose, paranasal sinuses and skull base. Rhinol Suppl 2010;(06)01;22:1-143.

3. Jangard M, Hansson J, Ragnarsson-Olding B. Primary sinonasal malignant melanoma: a nationwide study of the Swedish population, 1960-2000. Rhinology 2013;51(1):2230.

4. Lombardi D, Bottazzoli M, Turri-Zanoni M, Raffetti E, Villaret AB, Morassi ML, et al. Sinonasal mucosal melanoma: A 12-year experience of 58 cases. Head Neck 2016;38(Suppl 1):E1737-45

5. Lund VJ, Chisholm EJ, Howard DJ, Wei WI. Sinonasal malignant melanoma: an analysis of 115 cases assessing outcomes of surgery, postoperative radiotherapy and endoscopic resection. Rhinology 2012;50(2):203-210.
6. Gal TJ, Silver N, Huang B. Demographics and treatment trends in sinonasal mucosal melanoma. Laryngoscope 2011;121(9):20262033

7. Henderson MA, Burmeister $\mathrm{BH}$, Ainslie J, Fisher R, Di lulio J, Smithers BM, et al. Adjuvant lymph-node field radiotherapy versus observation only in patients with melanoma at high risk of further lymphnode field relapse after lymphadenectomy (ANZMTG 01.02/TROG 02.01): 6-year followup of a phase 3, randomised controlled trial. Lancet Oncol 2015;16(9):1049-1060.

8. Owens JM, Roberts DB, Myers JN. The role of postoperative adjuvant radiation therapy in the treatment of mucosal melanomas of the head and neck region. Arch Otolaryngol Head Neck Surg 2003;129(8):864-868.

9. Howard DJ, Lund VJ, Wei WI. Craniofacial resection for tumors of the nasal cavity and paranasal sinuses: a 25-year experience. Head Neck 2006;28(10):867-873.

10. Miglani A, Patel SH, Kosiorek HE, Hinni ML, Hayden RE, Lal D. Endoscopic resection of sinonasal mucosal melanoma has comparable outcomes to open approaches. Am J Rhinol Allergy 2017;31(3):200-204.

11. Koivunen $\mathrm{P}$, Makitie AA, Back L, Pukkila
M, Laranne J, Kinnunen I, et al. A national series of 244 sinonasal cancers in Finland in 1990-2004. Eur Arch Otorhinolaryngol 2012;269(2):615-621.

12. Ledderose GJ, Leunig A. Surgical management of recurrent sinonasal mucosal melanoma: endoscopic or transfacial resection. Eur Arch Otorhinolaryngol 2015;272(2):351356.

13. Swegal W, Koyfman S, Scharpf J, Sindwani R, Greskovich J, Borden E, et al. Endoscopic and open surgical approaches to locally advanced sinonasal melanoma: comparing the therapeutic benefits. JAMA Otolaryngol Head Neck Surg 2014;140(9):840-845.

14. Lund VJ, Wei WI. Endoscopic surgery for malignant sinonasal tumours: an eighteen year experience. Rhinology 2015;53(3):204211.

15. Roth TN, Gengler C, Huber GF, Holzmann D. Outcome of sinonasal melanoma: clinical experience and review of the literature. Head Neck 2010;32(10):1385-1392.

16. Jethanamest D, Vila PM, Sikora AG, Morris LGT. Predictors of survival in mucosal melanoma of the head and neck. Ann Surg Oncol 2011;18(10):2748-2756.

17. Koivunen P, Back L, Pukkila M, Laranne J, 
Kinnunen I, Grenman R, et al. Accuracy of the current TNM classification in predicting survival in patients with sinonasal mucosal melanoma. Laryngoscope 2012;122(8):1734-1738.

18. Samstein RM, Carvajal RD, Postow MA, Callahan MK, Shoushtari AN, Patel SG, et al. Localized sinonasal mucosal melanoma: Outcomes and associations with stage, radiotherapy, and positron emission tomography response. Head Neck 2016;38(9):13101317.

19. Gavriel H, McArthur G, Sizeland A Henderson M. Review: mucosal melanoma of the head and neck. Melanoma Res 2011;21(4):257-266.

20. Pittaka M, Kardamakis D, Spyropoulou D. Comparison of International Guidelines on Mucosal Melanoma of the Head and Neck: A Comprehensive Review of the Role of Radiation Therapy. In Vivo 2016;30(3):165170.

21. Suh JD, Ramakrishnan VR, Chi JJ, Palmer JN, Chiu AG. Outcomes and complications of endoscopic approaches for malignancies of the paranasal sinuses and anterior skull base. Ann Otol Rhinol Laryngol 2013;122(1):54-59.

22. Thiel A, Moza M, Kytola S, Orpana A, Jahkola T, Hernberg $M$, et al. Prospective immunohistochemical analysis of BRAF V600E mutation in melanoma. Hum Pathol 2015;46(2):169-175.

23. Griewank KG, Schilling B. Next-Generation Sequencing to Guide Treatment of Advanced Melanoma. A J Clin Dermatol 2017;18(3):303-310.

24. Shoushtari AN, Munhoz RR, Kuk D, Ott PA, Johnson DB, Tsai KK, et al. The efficacy of anti-PD-1 agents in acral and mucosal melanoma. Cancer 2016;122(21):3354-3362.

25. Weber J, Mandala M, Del Vecchio M, Gogas $\mathrm{HJ}$, Arance AM, Cowey CL, et al. Adjuvant Nivolumab versus Ipilimumab in Resected Stage III or IV Melanoma. N Engl J Med 2017;377(19):1824-1835.

26. Eggermont AMM, Blank CU, Mandala $M$, Long GV, Atkinson V, Dalle S, et al.
Adjuvant Pembrolizumab versus Placebo in Resected Stage III Melanoma. N Engl J Med 2018;378(19):1789-1801.

27. Schachter J, Ribas A, Long GV, Arance A Grob J, Mortier L, et al. Pembrolizumab versus ipilimumab for advanced melanoma: final overall survival results of a multicentre, randomised, open-label phase 3 study (KEYNOTE-006). Lancet 2017;390(10105):1853-1862.

M. Lundberg

Department of Otorhinolaryngology

Head and Neck Surgery

Helsinki University Hospital

Helsinki

Finland

E-mail: marie.lundberg@hus.fi

\section{ENDOSCOPIC SINUS AND SKULL BASE SURGERY}

$3^{\text {rd }}-5^{\text {th }}$ of June, 2019

Zurich - Switzerland

\section{RHINOPLASTY AND FACIAL PLASTIC SURGERY}

$6^{\text {th }}-7^{\text {th }}$ of June, 2019

Zurich - Switzerland

\section{COURSE DIRECTORS}

Prof. Dr. Daniel Simmen,

KD Dr. Hans Rudolf Briner

ORL-Zentrum, Klinik Hirslanden

Center for Rhinology, Skull Base Surgery and Facial Plastic Surgery, Zurich (CH)

\section{COURSE SECRETARY}

Sabrina Kuhnen | kuhnen@orl-zentrum.com

\section{COURSE LOCATION}

Institute of Anatomy, University of Zurich-Irchel Zurich - Switzerland

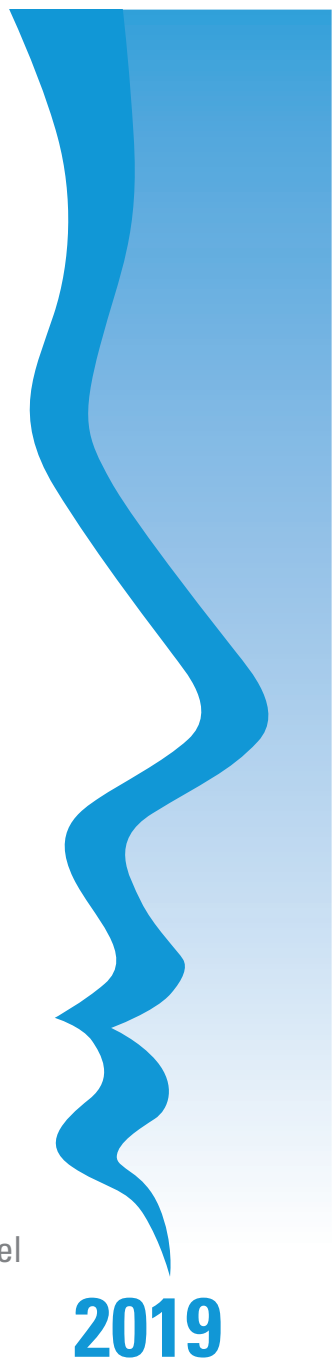

www.orl-zentrum.com 